\title{
QCD tests in DIS
}

\section{Olaf Behnke*}

DESY, Notkestrasse 85, Hamburg 22607, Germany

E-mail: obehnke@mail.desy.de

A review is given on the experimental tests of Quantum Chromo Dynamics (QCD) in Deep Inelastic Scattering (DIS). After an introduction, including a brief discussion of the future projects EIC and $\mathrm{LHeC}$, the latest results from HERA are presented. They comprise inclusive DIS, production of isolated photons, jets, heavy quarks and diffractive dijets. Theory calculations based on collinear or $k_{T}$ factorisation are compared to the data. Due to lack of space not all references can be provided; in particular for all references to the general theory and to specific model predictions the reader is referred to the cited experimental papers in which they can be found.

XXI International Workshop on Deep-Inelastic Scattering and Related Subject -DIS2013, 22-26 April 2013

Marseilles, France

\footnotetext{
${ }^{*}$ Speaker.
} 

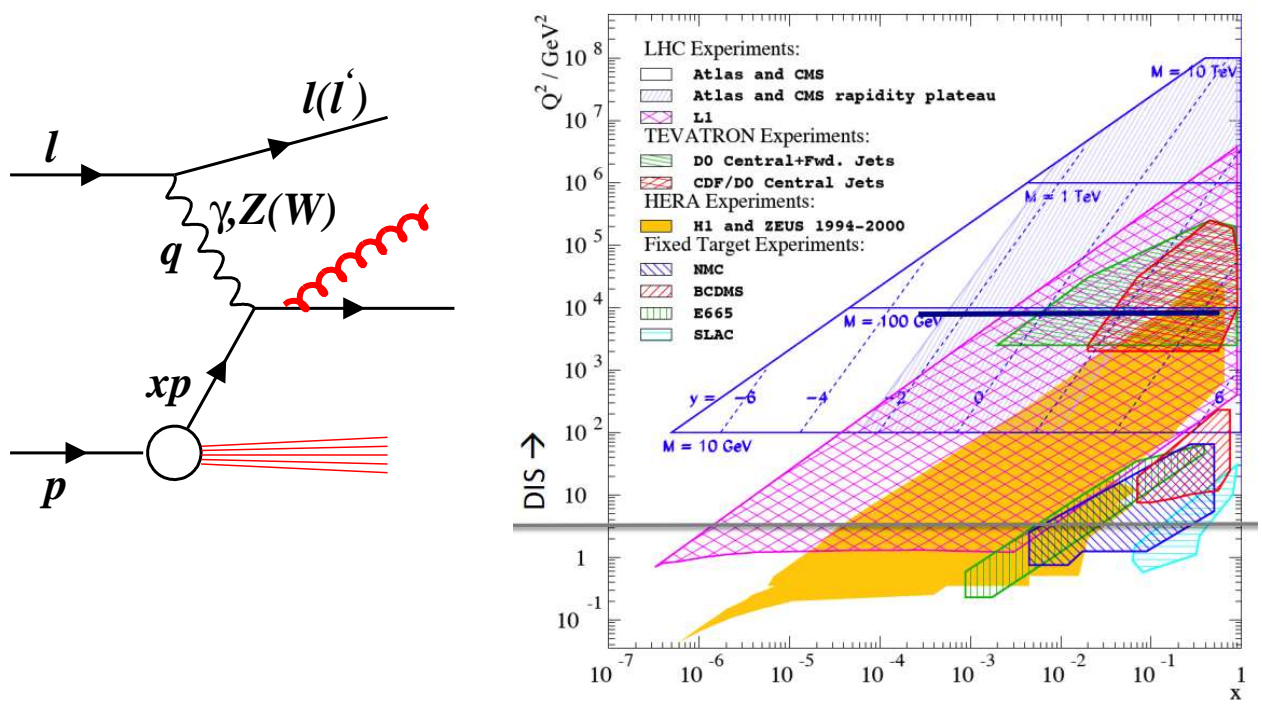

Figure 1: Left: Sketch of an exemplary higher order DIS process. Right:DIS kinematic plane of $Q^{2}$ and $x$ and coverage of various experiments.

\section{Introduction}

Since more than 40 years one studies in Deep Inelastic Scattering (DIS) simultaneously the nucleon structure and the strong interactions as represented by the theory of Quantum Chromo Dynamics (QCD). Figure 1 (left) shows a typical DIS process, as investigated e.g. at the ep collider HERA. A highly virtual gauge boson, either a photon, a $Z$ or a $W$ boson, is emitted from the lepton and knocks out a quark of the proton which is destroyed. One of the many possible higher order reactions of the strong interaction takes places: a hard gluon is emitted from the struck quark. The basic kinematics of the DIS scattering is described by the following observables:

- $Q^{2}=-q^{2}$, the negative of the squared four-momentum vector $q$ of the exchanged gauge boson.

- the inelasticity $y=q p / k p$, with $k$ and $p$ denoting the four-momentum vectors of the incident lepton and proton, respectively.

- the Bjorken $x$, defined as $x=Q^{2} / 2 p q$. In the quark parton model $x$ specifies the longitudinal momentum fraction of the proton carried by the quark that is struck by the gauge boson.

The three variables are constrained by the relation $Q^{2}=x y s$, where $s$ denotes the fixed squared centre-of-mass energy of the lepton-proton system.

Figure 1 (right) shows the kinematic plane in $Q^{2}$ and $x$, covered by various experiments in the past, present and future. The DIS region is defined by $Q^{2} \geq$ few $\mathrm{GeV}^{2}$, while the photoproduction region (exchange of quasi-real photons) is defined by $Q^{2}<1 \mathrm{GeV}^{2}$. Decades ago fixed target experiments started the exploration of the nucleon structure from the region of large $x$ and small $Q^{2}$. The H1 and ZEUS experiments at the HERA collider extended the phase space to the region of smallest $x \sim 0.000001$ and highest $Q^{2} \sim 40000 \mathrm{GeV}^{2}$. The coverage of the central detector experiments at the $p \bar{p}$ collider TEVATRON and the $p p$ collider LHC is also indicated in Figure 1. It 
overlaps with HERA; this makes the proton structure information from HERA so crucial for obtaining predictions for many important reactions at the two hadron colliders (e.g. Higgs production at the LHC). For the future a new generation of DIS experiments is planned. Currently proposals for two complementary facilities are at the center of attention:

- In the US EIC project [1], the idea is to build a very high luminosity electron ion collider which has a centre of mass-energy some (not large) factor below HERA. Polarised electron and ion beams are a key aspect of the project. One of the physics highlights is the 3D study of the nucleon structure and another one is the investigation of the nucleon spin structure. For a detailed discussion of the physics prospects see the talk by M. Stratmann [2].

- In the european $\mathrm{LHeC}$ project [3], electrons from a newly built machine with an energy of the order of 50-150 GeV are collided with protons (or heavy ions) from the LHC. The high beam energies enable a huge phase space extension compared to HERA (see Figure 1) reaching unprecedented low $x$ values and high $Q^{2}$ values. With anticipated luminosities of up to $10^{34} \mathrm{~cm}^{-2} \mathrm{~s}^{-1}$, orders of magnitudes larger than at HERA, the LHeC is the ultimate future high energy frontier DIS machine. Due to the high energy the electroweak interactions are extremely enhanced at the $\mathrm{LHeC}$; this is very beneficial for both the clean investigation of the nucleon structure and for precision tests of the strong and the electroweak interactions. A project status report is given at this conference by N. Armesto [4].

In the following we will discuss some selected highlights of new results from the H1 and ZEUS experiments at HERA, starting with inclusive and moving to more exclusive reactions. As a reminder, $\mathrm{H} 1$ and ZEUS collected each about $0.5 \mathrm{fb}^{-1}$ of $e p$ collisions; the first quarter was taken in the HERA I period and the other three in the HERA II period, where the lepton beams were polarised. About half of the data were taken with $e^{+} p$ collisions and the other with $e^{-} p$ collisions.

\section{Inclusive DIS}

Inclusive DIS means to count every DIS event in a given region of $Q^{2}$ and $x$. In general the DIS process is described in collinear factorisation by three connected pieces (see Figure 1 (left)): the exchange of an electroweak gauge boson, the hard scattering perturbative QCD (pQCD) process and the non perturbative Parton Distribution Functions (PDF) of the proton as represented by some parametrisations at a start scale $Q_{0}$ and evolved with the DGLAP evolution to the scale of the hard interaction. Usually one fixes one or two parts in the calculation and "tests" the remaining part(s): fixing the electroweak and perturbative QCD pieces one can determine the PDFs, as discussed in the talk by V. Radescu [5]; fixing the electroweak part one can test perturbative QCD convoluted with PDFs - this is the main talk topic, discussed from section 2.2 onwards. For the next subsection we turn the logic around and fix the PDFs and perturbative QCD and test the electroweak part.

\subsection{Electroweak tests}

Recently the H1 and ZEUS experiments completed their inclusive DIS analyses by releasing the full high $Q^{2}$ data sets taken in the HERA II period. The H1 publication [6] contains the finalised inclusive $e^{ \pm} p$ Neutral current(NC) and Charged Current (CC) data. The ZEUS paper [7] covers 

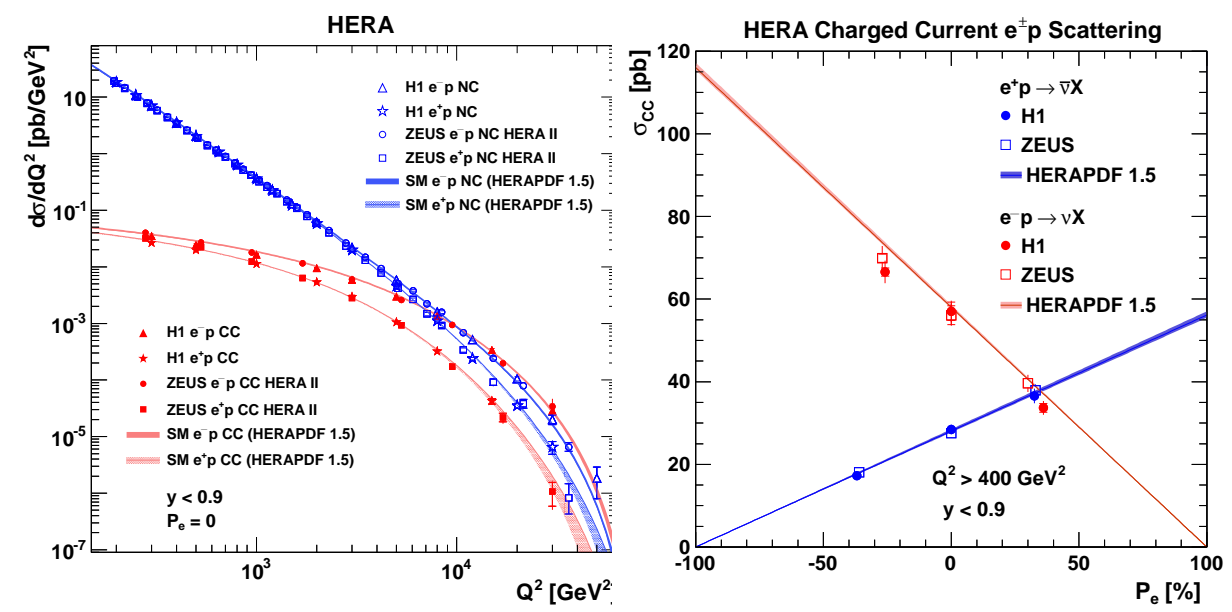

Figure 2: Left: Electroweak unification at HERA, as manifested in the $Q^{2}$ dependence of Neutral and Charged Currents. Right: Dependence of the total Charged Current cross section on the lepton beam longitudinal polarisation. The figures are available at [8].

the last missing high $Q^{2}$ data set from ZEUS, the $e^{+} p$ NC data. Figure 2 (left) shows the test of electroweak unification based on the complete data sets from both experiments. At low $Q^{2}$ the $\mathrm{NC}$ cross sections are much higher than the $\mathrm{CC}$ cross sections. Here the electromagnetic photon exchange dominates the $\mathrm{NC}$ reaction compared to the exchange of the weak $Z$ boson and it is also much stronger than the $\mathrm{CC}$ reaction mediated only by the weak $W$ boson. However, when $Q^{2}$ approaches the squared $Z$ and $W$ boson masses, the relative suppression of $Z$ and $W$ exchanges vanishes and the $\mathrm{NC}$ and $\mathrm{CC}$ cross section curves "merge". This shows that the electromagnetic and weak force unite and behave as one single force.

Figure 2 (right) shows the H1 and ZEUS inclusive CC cross sections as a function of the lepton beam polarisation $P_{e}$. According to the Standard Model (SM), the cross sections should depend linearly on the polarisation and vanish for a purely righthanded electron beam $\left(P_{e}=100 \%\right)$ and a purely lefthanded positron beam $\left(P_{e}=-100 \%\right)$. The data are in excellent agreement with the SM. As shown e.g. by $\mathrm{H} 1$ for their data [6], the results can be used to obtain lower limits on masses of hypothetical $W$ bosons, mediating charged currents for right handed fermions, of the order of $200 \mathrm{GeV}$.

\subsection{Perturbative QCD and PDF tests}

Figure 3 (left) shows the finalised H1 results [6] on inclusive $e^{ \pm} p$ NC DIS data, using a large fraction of all available data. The reduced cross section is measured as a function of $Q^{2}$ for various values of $x$. The data were obtained from a weighted averaging of the HERA II high $Q^{2}$ inclusive DIS data, their HERA I counterparts and also the HERA I low $Q^{2}$ data. An excellent experimental precision of about $1.5 \%$ has been reached for a large region $Q^{2}<500 \mathrm{GeV}^{2}$. In an Next-To-Leading Order (NLO) QCD analysis based on the DGLAP evolution equations, PDFs were fitted to the data. The fit function from the resulting PDF set "H1PDF2012", is also depicted in Figure 3. It provides a reasonable description of the data in the whole kinematic range. It was often hypothesised that one might find a prominent breakdown of the NLO DGLAP fit description at low $Q^{2}$ and low $x$ due to uncontrollable higher order QCD effects, but there is at least no blatant evidence from this plot. 

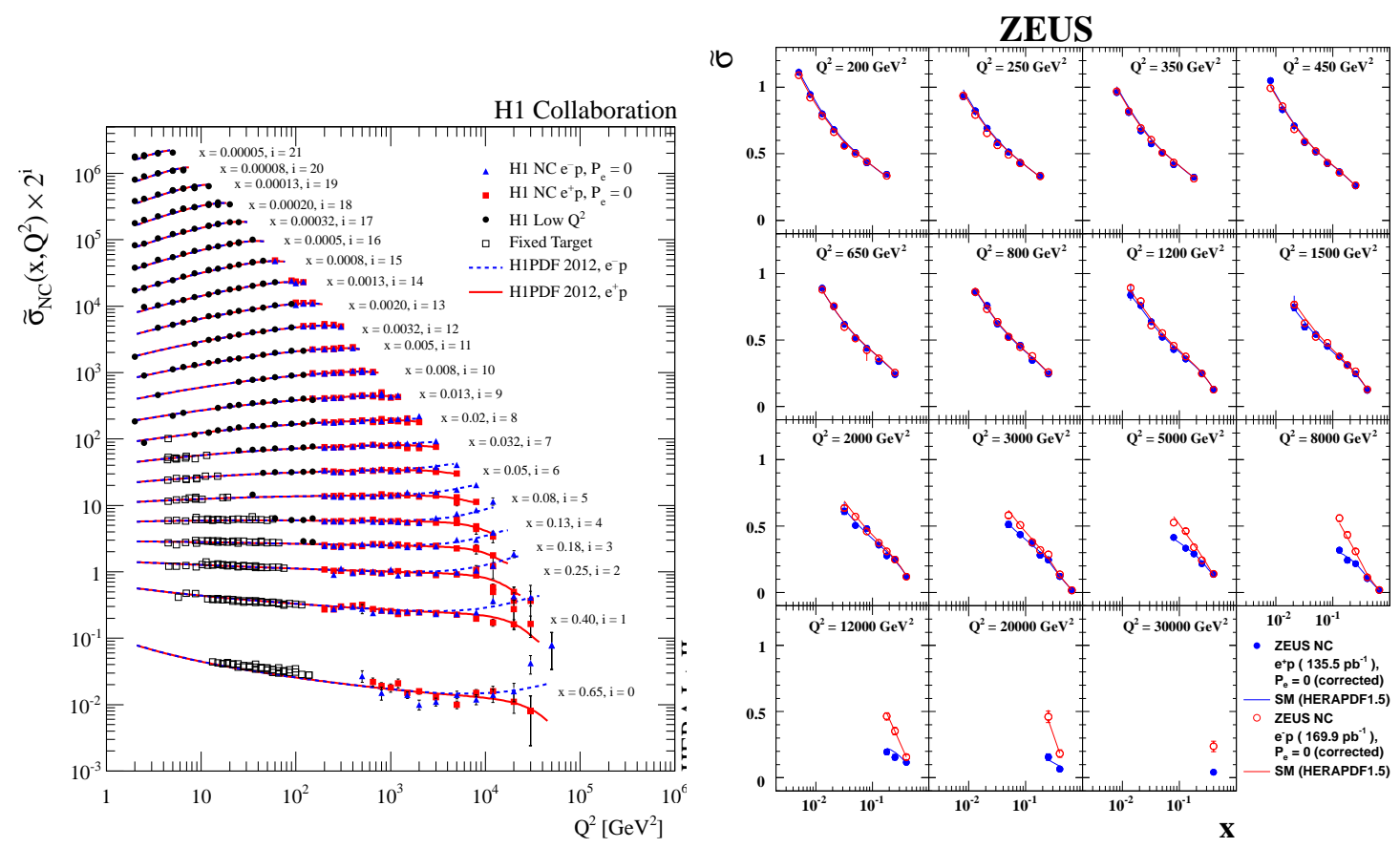

Figure 3: Recent Neutral Current reduced cross section measurements from H1 (left) and ZEUS (right).

Figure 3 (right) shows a similar new measurement of the $e^{ \pm} p$ NC reduced cross sections by ZEUS [7], using the HERA II high $Q^{2}$ data sets. Here the data are plotted as a function of $x$ for various fixed values of $Q^{2}$. A very good experimental precision is reached of about $1.5 \%$ in some lower $Q^{2}$ regions. Also shown is the prediction using the PDF set "HERAPDF1.5", which was obtained from an NLO DGLAP fit to specific subsets of the H1 and ZEUS inclusive data. As in the H1 case, the data are described well by the fit.

In the future it is planned to combine the finalised/new high $Q^{2}$ HERA II inclusive DIS data from $\mathrm{H} 1$ and ZEUS together with the HERA I combined inclusive data in order to obtain the best available HERA data set to be used for QCD analyses and PDF determinations.

\section{Isolated photon production}

Figure 4 shows (left) one of the leading order (LO) diagrams for the radiation of real photons at HERA. The photon emission is not affected by hadronisation and thus provides a direct probe of the parton dynamics of the hard scattering. Furthermore, the photon emissions can provide flavour information on the proton PDFs, since the photon couples stronger to $u$-type than to $d$-type quarks. ZEUS released recently a preliminary measurement [9] of the photoproduction of isolated photons with and without accompanying jets. The photons were required to be isolated and identified by using a shower shape observable. Figure 4 (middle) presents the measured cross sections for events with a photon and an additional jet, as a function of the jet pseudorapidity. NLO QCD predictions based on collinear factorisation as well as a $k_{T}$ factorisation model calculation were compared to the data; while the NLO model describes the data well, the $k_{T}$ calculation predicts a somewhat flatter shape than is observed in the data. Figure 4 (right) shows the cross sections as function of the transverse energy of the photon. Again, the NLO calculation describes the data well; the $k_{T}$ 


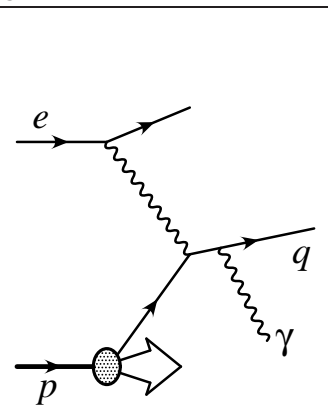

(b)
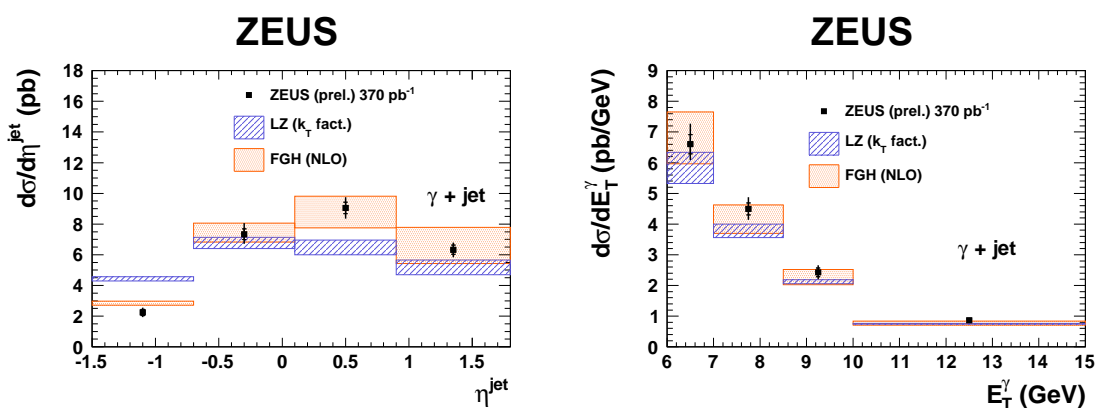

Figure 4: Left: Exemplary LO diagram for photon production at HERA. Middle/Right: Measured differential cross sections as functions of the rapidity of the accompanying jet in the event and the photon transverse energy, compared to two predictions.

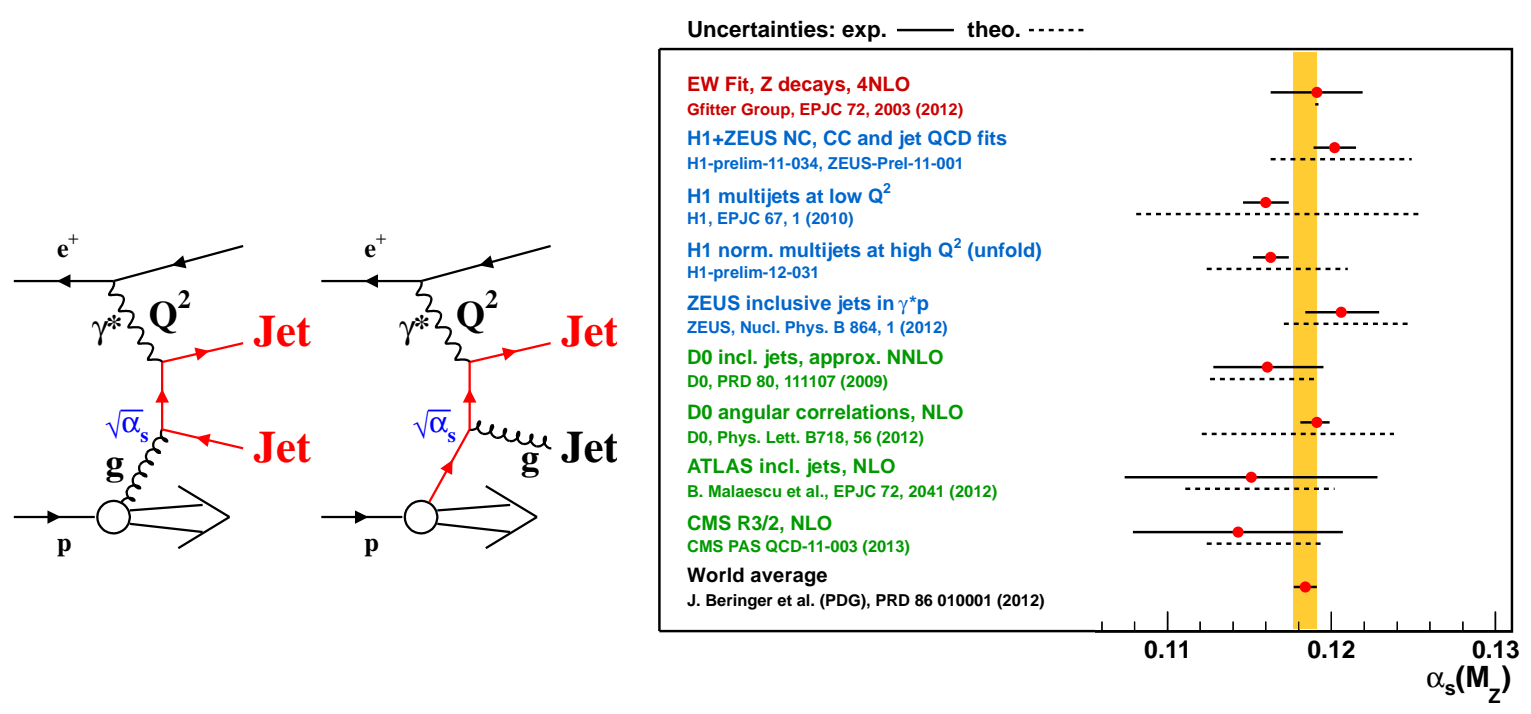

Figure 5: Left: Exemplary leading order diagrams for jet production in the $\gamma^{*} p$ frame. Right: Selected recent measurements of $\alpha_{S}\left(M_{Z}\right)$.

calculation is slightly too low in normalisation. Looking also at other differential cross sections the general picture emerges that both predictions provide a fair to reasonable prediction, but leaving room for improvement.

\section{Jets and $\alpha_{s}$}

Jet production at HERA is a classical tool to investigate the proton structure as well as to measure the strong coupling constant $\alpha_{s}$. Two exemplary leading order diagrams for jet production in the $\gamma^{*} p$ frame at HERA are shown in Figure 5 (left). The fact that both gluon and quark initiated processes occur, enables to separate the gluon density in the proton from the determination of $\alpha_{s}$. A compilation [10] of selected recent determinations of $\alpha_{s}\left(M_{Z}\right)$ is presented in Figure 5 (right).

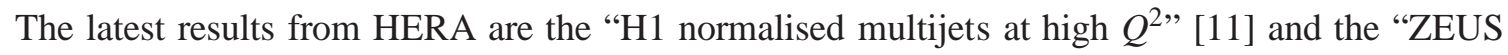
inclusive jets in photoproduction" [12]; both measurements were presented in detail at the previous DIS conference. The HERA jet based determinations of $\alpha_{s}\left(M_{Z}\right)$ have a very good experimental precision but suffer from the relatively large theory uncertainties; to date only NLO calculations are available and the higher order uncertainties estimated from scale variations are large. Several jet based measurements from TEVATRON and LHC are also shown in the Figure; they also suffer 
from large NLO theory uncertainties. A recent electroweak fit to $Z$ hadronic decays at LEP, using complete $o\left(\alpha_{s}^{4}\right)$ corrections is shown on the top and has the smallest theory uncertainty. All shown determinations agree well with a world average result, presented at the bottom; among various other types of measurements also lattice QCD based results have been used as input for this average.

\section{Heavy quark production}

The heavy charm and beauty quarks are produced at HERA mainly by boson gluon fusion (BGF) processes $\gamma g \rightarrow c \bar{c}$ or $b \bar{b}$. The leading order diagram is the same as the one in Figure 5 (left), but restricting the outgoing quark lines to charm or beauty quarks. Due to the large gluon density in the proton, the BGF processes gives large contributions to DIS; charm production alone accounts at low $x$ and high $Q^{2}$ for up to $\sim 35 \%$ of the inclusive DIS cross section. Furthermore, the process provides direct sensitivity to the gluon density in the proton. There are different schemes to treat the heavy flavour production (for references consult [13].) At low $Q^{2}$, that is $Q^{2} \sim m_{c}^{2}$, with $m_{c}$ denoting the charm quark mass, there is no doubt that the massive scheme is correct. The scheme is rigorous quantum field theory in which the masses of the heavy quarks are fully taken into account in every part of the calculation. In this scheme, charm and beauty quarks can only be dynamically produced in the hard interaction. The proton PDFs contain only light quarks and gluons and hence the scheme is also called Fixed-Flavour-Number Scheme (FFNS). However, at very high virtualities $Q^{2} \gg m_{c}^{2}$, it might be favourable to treat the charm quark as massless which is done in the Zero-Mass-Variable-Flavour-Number Scheme (ZMVNFS). In this scheme, the charm and beauty quarks appear above some kinematic thresholds also as massless sea quarks in the proton. The nice feature of this scheme is that it allows to resum to all orders certain logarithmic terms appearing in the perturbative calculation related to collinear gluon radiation from the heavy quark lines. The General-Mass-Variable-Flavour-Number Schemes (GMVFNS) make use of the best of "both worlds": at low $Q^{2}$ the massive scheme is used and at high $Q^{2}$ the massless scheme, with a suitable interpolation in the intermediate region. However, what is a suitable interpolation is debated for a long time as well as many other details of the treatment of mass dependent terms in pQCD; in fact there are numerous GMVFNS variants on the market and used by the various PDF fitter groups in the world.

\subsection{Open charm production in DIS}

Recently, H1 and ZEUS published the combination of charm production data in DIS [13]. The data, based on various tagging methods such as full reconstruction of $D^{*}$ mesons, $D^{+}$mesons, semileptonic decays, or inclusive secondary vertex tagging, have been combined at the level of the so called reduced cross sections. This cross section is related to the total charm production cross section in a region of $Q^{2}$ and $x$. In the combination the correlated systematics is fully taken into account. The combined data reach an (for charm production at HERA) unprecedented best precision of about $5 \%$ in certain phase space regions. Figure 6 (left) shows the obtained combined reduced cross sections as a function of $x$ for various values of $Q^{2}$. Also shown is the prediction based on the "HERAPDF1.5" PDF, using the GMVFNS in the "Robert Thorne standard" scheme variant (for proper references consult [13]). These PDFs were obtained from fitting only inclusive DIS data, without any knowledge of charm production data. The prediction describes the charm 

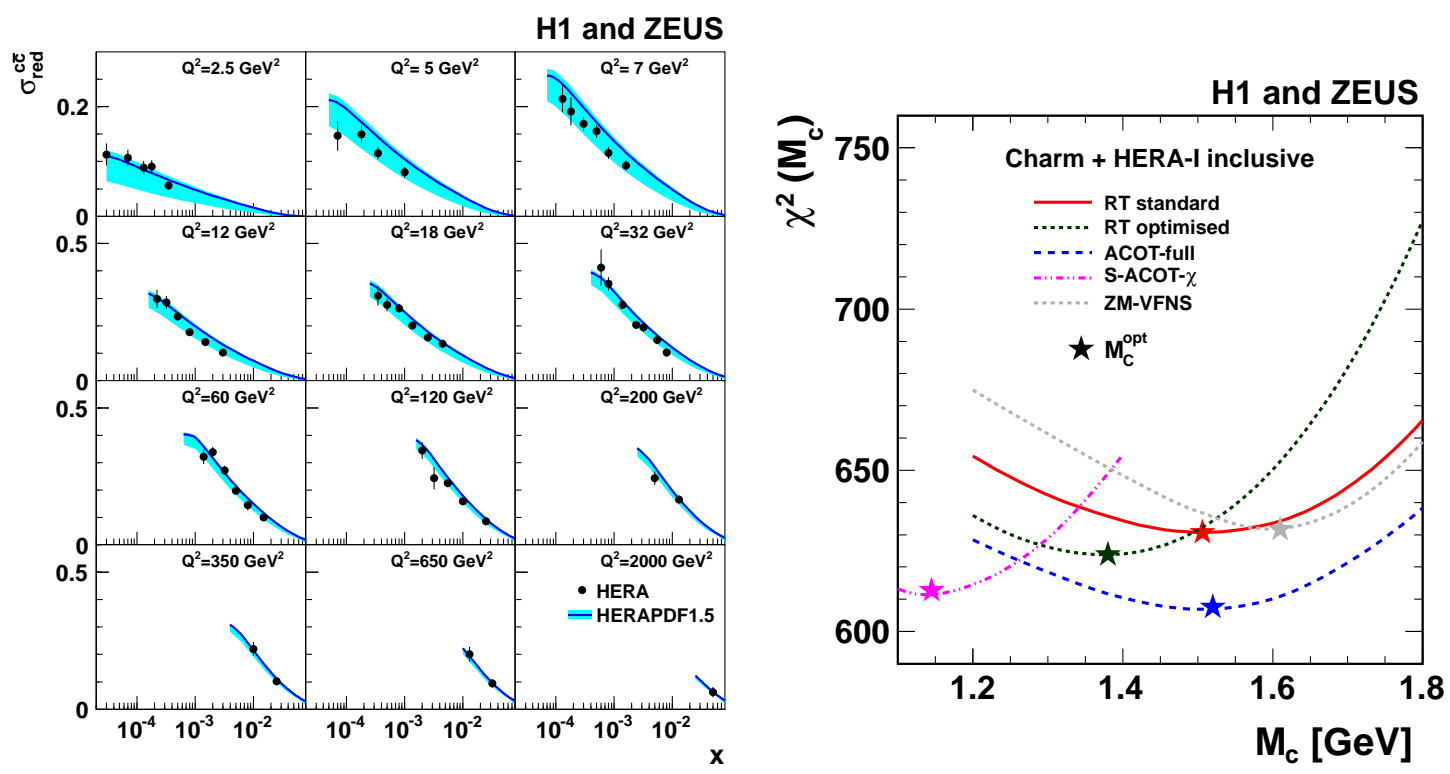

Figure 6: Left: Reduced cross sections for charm production in DIS. The HERA combined data are compared to a GMVFNS prediction. Right: $\chi^{2}$ of the PDF plus charm mass fit as a function of the charm mass parameter $M_{c}$ - for further details see the main text.

data reasonably well which is a triumph for the QCD collinear factorisation approach used in the calculations: the gluon density in HERAPDF1.5, obtained from the scaling violations of inclusive DIS, is in agreement with the gluon density that is needed to describe the charm production data. The not so nice thing is the large theory uncertainty, as indicated by the error band, which is dominated by variations of the charm quark mass. Turning the tables, a simultaneous fit of PDFs plus the charm quark pole mass was performed in [13]. Both the HERA combined charm data and combined HERA I inclusive DIS data were used as input and various GMVFNS variants were tried out. Figure 6 (right) shows a $\chi^{2}$ scan of this fit as a function of the charm mass parameter $M_{C}$. It can be seen that the fitted $M_{c}$ values (called optimal mass " $M_{c}^{o p t}$ " in the plot) differ considerably between the various GMVFNS variants (e.g. "RT standard", "S-ACOT- $\chi$ "). In fact, when using a fixed value $M_{c}=1.4 \mathrm{GeV}$, some of the schemes fail to describe (not shown here) the combined charm data in the lower $Q^{2}$ regions, while when using the optimal masses most of the schemes give a good description. What can we learn from this? The various GMVFNS interpolate differently between the massive and massless schemes and this leads to a different quality of the charm data description for a fixed charm mass; these deficiencies can be compensated by using the $M_{c}^{\text {opt }}$ values. This has even broader ramifications as discussed in the talk by V. Radescu [5]: Using the specific $M_{c}^{o p t}$ value for each scheme stabilises the PDF mixture of the various quark flavours and of the gluon; in turn this stabilises predictions for $W$ and $Z$ production at the LHC which depend crucially on the flavour mixture.

The massive scheme predictions (not shown here) which are available at NLO plus partial NNLO, provide the best description of the HERA charm combined data. A simultaneous fit of PDFs and of the running charm quark mass has been performed with this scheme, yielding a value $m_{c}\left(m_{c}\right)=1.26 \pm 0.06 ; \mathrm{GeV}$, which is consistent with the world average value $m_{c}\left(m_{c}\right)=1.275 \pm$ $0.025 \mathrm{GeV}$ [14]. For a detailed discussion of all the interesting results from [13] the reader is referred to the talk by A. Geiser [15]. 

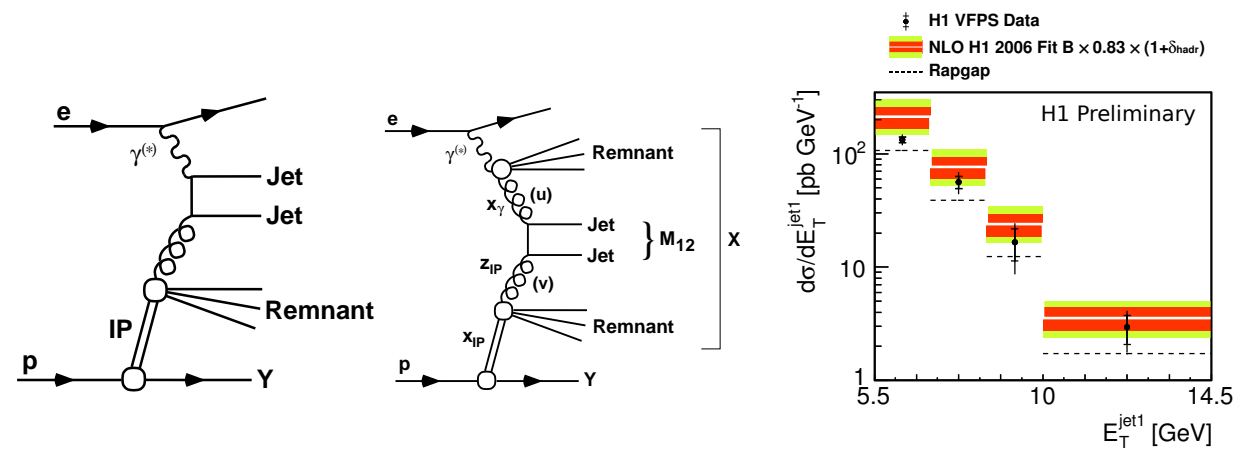

Figure 7: Left: Direct- and resolved-photon diagrams for diffractive dijet photoproduction at HERA. Right: Measured cross sections of VFPS tagged diffractive photoproduction dijet data as a function of the transverse energy of the leading jet.

At this conference two further brand new publications on charm production in DIS have been presented by ZEUS, based on the full reconstruction of $D^{*}$ mesons [16] or $D^{+}$mesons [17]. The data have good precision and are consistent with the HERA combined charm data; in the future they can be used to improve the combination.

Finally a general remark: the existing measurements of open charm and beauty quarks at HERA in DIS and also in photoproduction are described over the whole kinematic phase space, from smallest to largest transverse momenta or $Q^{2}$, by the NLO massive scheme calculations, so one could say that the massive scheme prevails at HERA.

\section{Diffractive dijets in photoproduction}

H1 has presented at this conference a new (preliminary) measurement [18] of diffractive dijet photoproduction, tagging the elastically scattered proton in their "Very Forward Proton Spectrometer" (VFPS). Figure 7 (left to middle) shows diagrams of the process in the resolved pomeron model (the scattered outgoing proton is labelled here as $Y$ ). One of the specific interests in photoproduction is the expected presence of resolved photon processes (the second diagram). These processes resemble hadron-hadron collisions and could shed light on the mechanism of the strong suppression of diffractive dijet production that was observed at the TEVATRON [19] and very recently also at the LHC [20] This suppression is with respect to predictions based on the resolved pomeron model in which QCD factorisation is assumed: this means that the densities of partons from the pomeron entering the hard interaction (in the diagrams shown in the Figure it is a gluon) are assumed to be universal and can be determined e.g. from inclusive diffractive DIS. One explanation for the observed suppression is is a rescattering of the proton remnant with the elastically scattered proton. In HERA such rescattering could also take place between the resolved photon remnant and the escaping proton.

Figure 7 (right) shows the differential cross sections measured by $\mathrm{H} 1$ [18] as a function of the leading jet transverse energy. The data show a relative suppression with respect to the factorisation based NLO prediction by a factor $\sim 0.7$, however the uncertainties are too large to draw strong conclusions. In general the obtained picture of factorisation breaking in diffractive photoproduction of dijets at HERA remains somewhat inconclusive, in particular there is no hint for a larger suppression in resolved photon enhanced samples. 


\section{Conclusions}

Six years after the end of the HERA data taking there is still a continuous flow of new QCD precision results from the H1 and ZEUS experiments. Recently both experiments completed (and published) the analysis of the full high $Q^{2}$ data sets taken in the HERA II period. As a result, DIS data sets are now available spanning a huge phase space from smallest to highest $Q^{2}$ and $x$, with high precision up to $Q^{2}$ of a few $1000 \mathrm{GeV}^{2}$. Jet measurements from HERA have also reached a very high precision, making it possible to determine $\alpha_{s}\left(M_{Z}\right)$ with an experimental uncertainty of $1-2 \%$. NNLO calculations are urgently needed to provide an adequate small theory uncertainty. The combination of $\mathrm{H} 1$ and ZEUS charm data in DIS provides a data set with best precisions of $\sim 5 \%$. These data are a unique testing ground for the treatment of heavy quark mass dependent terms in pQCD. H1 presented new results on diffractive dijets using their very forward proton spectrometer, adding information on the question of factorisation breaking in photoproduction. Many more new intriguing HERA results on the production of specific final states are available which could not be covered here: $Z$ bosons, beauty quarks, $J / \psi$ mesons, charged particles, and others. The interested reader can find a detailed discussion of all of them in other proceeding articles of this conference.

In the future the complementary $\mathrm{LHeC}$ and EIC projects, if they are realised, would start a new era of DIS with unprecedented opportunities for precision tests of QCD.

\section{References}

[1] EIC White Paper, arXiv:1212.1701v2 [nucl-ex].

[2] M. Stratmann, these proceedings.

[3] LHeC Study Group, J. Phys. G 39 (2012) 075001 [arXiv:1206.2913 [physics.acc-ph]].

[4] N. Armesto, these proceedings.

[5] V. Radescu, these proceedings.

[6] H1 Collaboration, JHEP 1209 (2012) 061 [arXiv:1206.7007 [hep-ex]].

[7] ZEUS Collaboration, Phys. Rev. D 87 (2013) 052014 [arXiv:1208.6138 [hep-ex]].

[8] HERA combined results webpages: wWw. desy.de/h1zeus/combined_results/index.php?do=proton_structure.

[9] ZEUS Collaboration, ZEUS-prel-13-001.

[10] R. Kogler, private communication.

[11] H1 Collaboration, H1prelim-12-031.

[12] ZEUS Collaboration, Nucl. Phys. B 864 (2012) 1 [arXiv:1205.6153 [hep-ex]].

[13] H1 and ZEUS Collaborations, Eur. Phys. J. C 73 (2013) 2311 [arXiv:1211.1182 [hep-ex]].

[14] K. Nakamura et al. [Particle Data Group Collaboration], J. Phys. G 37 (2010) 075021.

[15] A. Geiser, these proceedings.

[16] ZEUS Collaboration, JHEP 1305 (2013) 097 [arXiv:1303.6578 [hep-ex]].

[17] I. Abt et al. [ZEUS Collaboration], JHEP 1305 (2013) 023 [arXiv:1302.5058 [hep-ex]].

[18] H1 Collaboration, H1 prelim-13-011.

[19] T. Affolder et al. [CDF Collaboration], Phys. Rev. Lett. 84 (2000) 5043.

[20] S. Chatrchyan et al. [CMS Collaboration], Phys. Rev. D 87 (2013) 012006 [arXiv:1209.1805 [hep-ex]]. 